\title{
On Fuzzy Spheres in Fuzzy Lobachevsky Space and its Retractions
}

\author{
A. E. El-Ahmady ${ }^{1,2^{*}}$ and K. Al-Onemi ${ }^{3}$ \\ 1,2 Mathematics Department, Faculty of Science, Taibah University, Madinah, Saudi Arabia; a_elahmady@hotmail.com \\ ${ }^{3}$ Mathematics Department, Faculty of Science, Tanta University, Tanta, Egypt
}

\begin{abstract}
This paper attempts to introduce and study new connection between fuzzy retractions, fuzzy foldings and fuzzy deformation retracts of open fuzzy spheres in fuzzy Lobachevsky space and fuzzy open ball in fuzzy Euclidean space. Types of fuzzy foldings and fuzzy deformation retracts of fuzzy open sphere are discussed. Types of minimal fuzzy retractions of fuzzy open sphere are presented. The fuzzy foldings of fuzzy open sphere is deduced, also the limit of this folding is obtained. The connection between fuzzy foldings and fuzzy central projection map is achieved; also the connection between fuzzy retractions and fuzzy exponential map is discussed. Some applications are presented.
\end{abstract}

Keywords: Fuzzy Retractions, Fuzzy Foldings, Fuzzy Deformation Retracts, Central Projection Map, Fuzzy Open Sphere in Fuzzy Lobachevsky Space.

\section{Introduction and Background}

Lobachevsky space represents one of the most intriguing and emblematic discoveries in the history of geometry. Although if it were introduced for a purely geometrical purpose, they came into prominence in many branches of mathematics and physics. This association with applied science and geometry generated synergistic effect: applied science gave relevance to Lobachevsky space and Lobachevsky space. Allowed formalizing practical problems $[13,20-23,28,30]$.

At each point $p$ of a complete Riemannian manifold $M$, we define a mapping of the tangent space $T_{p}(M)$ at $p$ onto $M$ in the following manner. If $X$ is a tangent vector at $p$, we draw a geodesic $g(t)$ starting at $p$ in the direction of $X$. If $X$ has length $\alpha$, then we map $X$ into the point $g(\alpha)$ of the geodesic. We denote this mapping by $\exp _{p}: T_{p}(M) \rightarrow M$, the map $\exp _{p}$ is everywhere $C^{\infty}$ and in a neighborhood of $p$ in $M$, and it is a diffeomorphism $[11,19]$.

As is well known, the theory of retractions is always one of interesting topics in Euclidian and Non-Euclidian spaces and it has been investigated from the various viewpoints by many branches of topology and differential geometry $[4,11,20,21,26,29]$.

There are many diverse applications of certain phenomena for which it is impossible to get relevant data. It may not be possible to measure essential parameters of a process such as the temperature inside molten glass or the homogeneity of a mixture inside some tanks. The required measurement scale may not exist at all, such as in the case of evaluation of offensive smells, evaluating the taste of foods or medical diagnoses by touching $[9,12,24,27]$.

The aim of the present paper is to describe the above phenomena geometrically, specifically concerned with the study of the new types of fuzzy retractions, fuzzy deformation retracts and fuzzy folding of fuzzy open sphere $\overline{\bar{S}^{n}-(\gamma)}$ in fuzzy Lobachevsky space as presented by El-Ahmady [1-12].

A fuzzy manifold is manifold which has a physical character. This character is represented by the density function $\mu$, where $\mu \in[0,1][7,8]$.

* Corresponding author:

A. E. El-Ahmady (a_elahmady@hotmail.com) 
A fuzzy subset $(\underset{\sim}{A}, \mu)$ of a fuzzy manifold $(\underset{\sim}{M}, \mu)$ is called a fuzzy retraction of $(\underset{\sim}{M}, \mu)$ if there exist a continuous map $\tilde{r}:(\underset{\sim}{M}, \mu) \longrightarrow(\underset{\sim}{A}, \mu)$ such that $\tilde{r}(a, \mu(a))=(a, \mu(a)), \forall$ $\mathrm{a} \in \underset{\sim}{A}, \mu \in[0, \widetilde{1}][1,5]$.

A fuzzy subset $(\underset{\sim}{\mathbb{M}}, \tilde{\mu})$ of a fuzzy manifold $(\underset{\sim}{M}, \mu)$ is called a fuzzy deformation retract if there exists a fuzzy retraction $\tilde{r}:(\underset{\sim}{\mathcal{M}}, \mu) \longrightarrow(\underset{\sim}{\bar{M}}, \tilde{\mu})$ and a fuzzy homotopy $\tilde{F}:(\underset{\sim}{M}, \mu) \times I \rightarrow(\underset{\sim}{M}, \mu)[11,25,26]$ such that

$$
\left.\begin{array}{l}
\tilde{F}((x, \mu), 0)=(x, \mu) \\
\tilde{F}((x, \mu), 1)=\tilde{r}(x, \mu)
\end{array}\right\} x \in \underset{\sim}{M}
$$

$\tilde{F}((a, \mu), t)=(a, \mu), \forall(a, \mu) \in \underset{\sim}{\mathcal{M}}, t \in I, \mu \in[0,1]$. Where $\tilde{r}(x, \mu)$ is the retraction mentioned above.

Topological folding of fuzzy sphere in fuzzy Lobachevsky space is reported $[2,3,10]$. A map $\widetilde{\mathfrak{I}}: \widetilde{S^{n}} \rightarrow \widetilde{S^{n}}$, is said to be an isometric folding of fuzzy sphere in fuzzy Lobachevsky space into itself iff for any piecewise fuzzy geodesic path $\gamma: J \rightarrow \widetilde{S^{n}}$ the induced path $\widetilde{\mathfrak{I}} \circ \gamma: J \rightarrow \widetilde{\mathrm{S}^{\mathrm{n}}}$ is a piecewise fuzzy geodesic and of the same length as $\gamma$, where $\mathrm{J}=[0,1]$. If $\widetilde{\mathfrak{I}}$ does not preserve lengths, then $\widetilde{\mathfrak{I}}$ is a topological folding of fuzzy sphere in fuzzy Lobachevsky space $[8,9,13-19]$.

The isofuzzy folding of $\bigcup \widetilde{M}_{i} \subseteq \widetilde{S^{n}}$ is a folding $\tilde{\mathfrak{I}}: \bigcup \tilde{M}_{i} \longrightarrow \bigcup \tilde{M}_{i}$ such that $\tilde{\mathfrak{I}}(\tilde{M})=\tilde{M}$ and any $\tilde{M}_{i}$ belong to the upper hypermanifolds $\exists \tilde{M}_{j}$ down $\tilde{M}$ such that $\mu_{i}=\mu_{j}$ for every corresponding points i.e. $\mu\left(a_{i}\right)=\mu\left(a_{j}\right)$ [6] (Figure 1).

\section{Main Results}

Theorem 1. Let $\tilde{S}^{n} \subset \widetilde{L}^{n+1}$ be a fuzzy sphere in a fuzzy Lobachevsky space which is homeomorphic to $\widetilde{D}^{n-1} \subset \widetilde{R}^{n}, \widetilde{\mathfrak{I}}: \tilde{S}^{n} \rightarrow \tilde{S}^{n}$ be a fuzzy folding, then there is an induced fuzzy folding $\widetilde{\widetilde{J}} \widetilde{D}^{n-1} \rightarrow \widetilde{D}^{n-1}$ such that the following diagram is commutative.

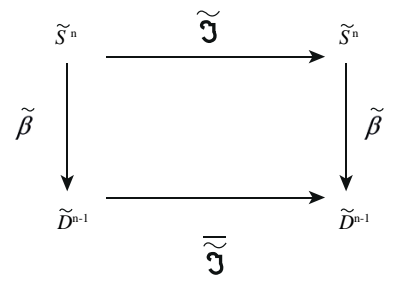

Proof. Let $\widetilde{\mathfrak{I}}: \tilde{S}^{n} \rightarrow \tilde{S}^{n}$ be a fuzzy folding. Then under the central projection map $\tilde{\beta}, \tilde{\beta}: \tilde{S}^{n} \rightarrow \widetilde{D}^{n-1}$ there is an induced fuzzy folding $\overline{\widetilde{I}}: \widetilde{D}^{n-1} \rightarrow \widetilde{D}^{n-1}$ such that $\tilde{\beta} \circ \widetilde{\mathfrak{I}}=\widetilde{\mathfrak{I}} \circ \tilde{\beta}$.

Theorem 2. Let $\tilde{S}^{n} \subset \widetilde{L}^{n+1}$ be a fuzzy sphere, homeomorphic to a fuzzy open ball $\widetilde{D}^{n-1} \subset \widetilde{R}^{n}$. Then, for any fuzzy

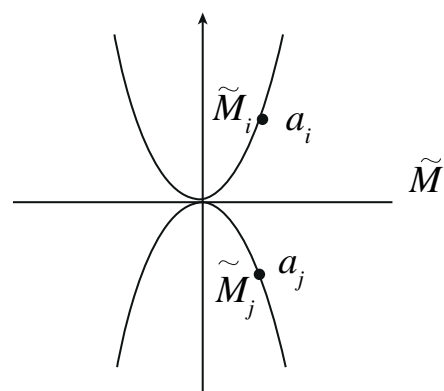

Figure 1. The isofuzzy folding of fuzzy manifold.

folding $\widetilde{\mathfrak{I}}: \tilde{S}^{n} \rightarrow \tilde{S}^{n}$, there are induced fuzzy foldings $\overline{\widetilde{I}}_{i}: \overline{\widetilde{D}}_{i}^{n-1} \rightarrow \overline{\widetilde{D}}_{i}^{n-1}, \widetilde{\mathfrak{I}}_{i}: \widetilde{\widetilde{D}}_{i}^{n-1} \rightarrow{\underline{D_{i}}}^{n-1}$.

Proof. Consider a fuzzy sphere $\tilde{S}^{n} \subset \widetilde{L}^{n+1}$, where the fuzzy point of $\tilde{S}^{n}$ have a different membership degree. Under the central projection map, any fuzzy on $\tilde{S}^{n}$ induced fuzzy system which will be fuzzy open balls $\overline{\widetilde{D}}_{i}^{n-1}, \widetilde{D}_{i}^{n-1}$. Also if $\widetilde{\mathfrak{I}}\left(\tilde{S}^{n}\right)=\tilde{S}^{n}$. Then the isofuzzy folding $\widetilde{\mathfrak{I}}_{i}: \widetilde{D}_{i}^{n-1} \rightarrow \overline{\mathrm{D}}_{i}{ }^{n-1}$.

Corollary 1. The relation between the fuzzy exponential map and fuzzy folding of a fuzzy sphere $\tilde{S}^{n} \subset \widetilde{L}^{n+1}$ discussed from the following commutative diagram.

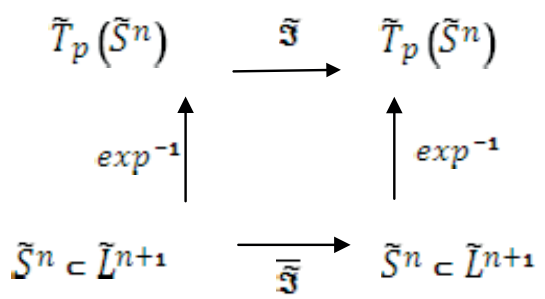

Proof. Since $\exp ^{-1}: \tilde{S}^{n} \rightarrow \widetilde{T}_{p}\left(\tilde{S}^{n}\right)$ be the fuzzy exponential map of $\tilde{S}^{n}$, the fuzzy folding of $\tilde{S}^{n}, \widetilde{T}_{p}\left(S^{n}\right)$, are given by $\widetilde{\mathfrak{I}}: \tilde{S}^{n} \subset \widetilde{L}^{n+1} \rightarrow \tilde{S}^{n} \subset \widetilde{L}^{n+1}, \widetilde{\mathfrak{I}}: \widetilde{T}_{p}\left(\tilde{S}^{n}\right) \rightarrow \widetilde{T}_{p}\left(\tilde{S}^{n}\right)$. Hence the following diagram is commutative, i.e. $\exp ^{-1} \circ \overline{\widetilde{\mathfrak{I}}}=\widetilde{\mathfrak{I}} \circ \exp ^{-1}$.

Theorem 3. The fuzzy folding of fuzzy sphere $\tilde{S}^{n}$ of fuzzy Lobachevsky space $\widetilde{L}^{n+1}$ is diffeomorphic to fuzzy folding of fuzzy open disk $\widetilde{D}(p, r)$ in $\widetilde{R}^{n}$.

Proof. Consider a fuzzy sphere $\tilde{S}^{n}$ in a fuzzy Lobachevsky space $\tilde{L}^{n+1}$ with fuzzification $\cup \tilde{\tilde{S}}_{i}^{n}, \cup \tilde{S}_{i}^{n}$. Let $\widetilde{\mathfrak{S}}: \cup \tilde{\tilde{S}}_{i}^{n} \rightarrow \cup \tilde{S}_{i}^{n}$ be the isofuzzy folding. Now, let us consider the central projection map $\tilde{\beta}: \tilde{S}^{n} \rightarrow \widetilde{R}^{n}$. The map $\widetilde{\beta}$ takes $\tilde{S}^{n}$ diffeomorphically to the fuzzy open disk $\widetilde{D}(p, r) \subset \widetilde{R}$, of center $p$ and radius $r=1$. Under the diffeomorphism between $\widetilde{L}^{n+1}$ and $\widetilde{R}^{n}$ there exists a fuzzy open disk $\widetilde{D}(p, r) \subset \widetilde{R}$ with fuzzification $\cup \widetilde{D}_{i}(p, r), \cup \widetilde{D}_{i}(p, r)$ and there is an induced isofuzzy folding $\beta, \widetilde{\mathfrak{J}} \cup \widetilde{\widetilde{D}}_{i}(p, r) \rightarrow \cup \underline{\mathbb{D}}_{i}(p, r)$. 
TheOREM 4. Let $\tilde{S}^{n} \subset \tilde{L}^{n+1}$ which is homeomorphic to $\tilde{D}^{n-1} \subset \tilde{R}^{n}$ and $\tilde{r}_{1}: \tilde{S}^{n} \rightarrow \tilde{S}^{n-1}$ be a fuzzy retraction map, then there is induced fuzzy retraction $\tilde{r}_{2}: \tilde{D}^{n-1} \rightarrow \tilde{D}^{n-2}$ such that the following diagram is commutative.

Proof. Let $\tilde{r}_{1}: \tilde{S}^{n} \subset \tilde{L}^{n+1} \rightarrow \tilde{S}^{n-1} \subset \widetilde{L}^{n+1}, \tilde{r}_{2}: \widetilde{D}^{n-1} \subset \widetilde{R}^{n} \rightarrow$ $\widetilde{D}^{n-2} \subset \widetilde{R}^{n}, \widetilde{D}^{n-1}, \widetilde{D}^{n-2}$ are opens ball in $\mathrm{n}$ - Euclidean space. Using the central projection map $\widetilde{\beta}_{1}, \widetilde{\beta}_{1}: \tilde{S}^{n} \subset \widetilde{L}^{n+1} \rightarrow \widetilde{D}^{n-1}$ $\subset \widetilde{R}^{n}, \widetilde{\beta}_{2}: \tilde{S}^{n-1} \subset \tilde{L}^{n+1} \rightarrow \tilde{D}^{n-2} \subset \tilde{R}^{n}$, then the following diagram is commutative

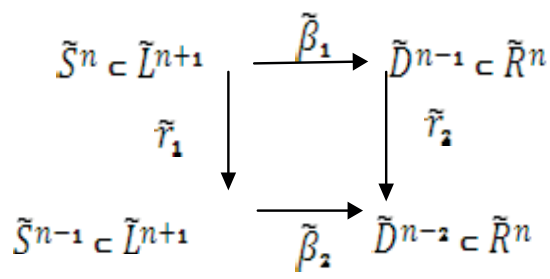

i.e. $\tilde{r}_{2} \circ \tilde{\beta}_{1}=\tilde{\beta}_{2} \circ \tilde{r}_{1}$

THEOREM 5. If $\tilde{r}_{i}: \exp ^{-1}\left(\tilde{S}_{i}^{n}\right) \subset \widetilde{L}^{n+1} \rightarrow T_{p}\left(\tilde{S}_{i}^{n}\right) \subset \tilde{L}^{n+1}, \tilde{r}_{i}: \tilde{S}^{n}$ $\subset \tilde{L}^{n+1} \rightarrow \tilde{S}_{i}^{n} \subset \tilde{L}^{n+1}$ and $\exp ^{-1}: \tilde{S}^{n} \rightarrow \widetilde{T}_{p}\left(\tilde{S}_{i}^{n}\right)$. Then $\exp ^{-1} \circ \tilde{r}_{i}=$ $\underline{\tilde{r}}_{i} \circ \exp ^{-1}$.

Proof. Consider the fuzzy exponential map $\exp ^{-1}$ : $\tilde{S}^{n} \rightarrow \widetilde{T}_{p}\left(\tilde{S}^{n}\right)$ and the fuzzy retractions be defined as $\tilde{r}_{\circ}: \tilde{S}^{n} \rightarrow \tilde{S}_{1}^{n}, \tilde{r}_{1}: \tilde{S}_{1}^{n} \rightarrow \tilde{S}_{2}^{n}, \ldots$, then there are induced commutative diagrams given by

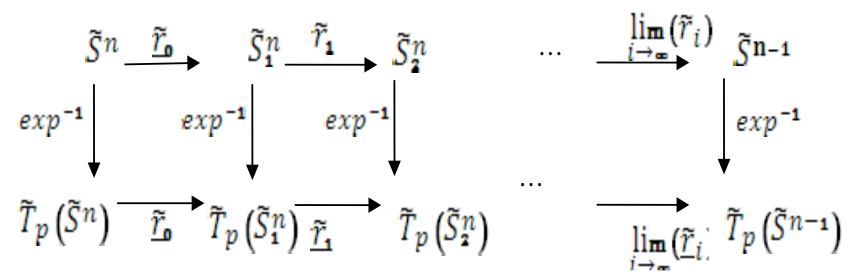

Such that $\exp ^{-1} \circ \tilde{r}_{i}=\tilde{\tilde{r}}_{i} \circ \exp ^{-1}$.

Theorem 6. Let $\tilde{S}^{n} \subseteq \widetilde{L}^{n+1}$ be a fuzzy sphere in a fuzzy Lobachevsky space $\widetilde{L}^{n+1}$ and $\widetilde{D}^{n-1}\left(p, r_{1}\right) \subset \widetilde{R}^{n}$ be a fuzzy open disk in n- Euclidean space $\widetilde{R}^{n}$. Then $\left(\tilde{f}_{i+1} \circ \tilde{r}_{i}=\underline{\tilde{r}}_{i} \circ \tilde{f}\right)=$ $: \widetilde{\beta}\left(\widetilde{\mathfrak{I}}_{i+1} \circ \tilde{r}_{i}=\widetilde{\mathfrak{I}}_{i} \circ \tilde{r}_{i}\right)$

Proof. Consider the fuzzy retraction $\tilde{S}^{n-1}, \tilde{S}^{n-2}, \ldots, \tilde{S}^{3}, \tilde{S}^{2}, \tilde{S}^{1}$ in a fuzzy sphere $\tilde{S}^{n}$ in a fuzzy Lobachevsky space $\tilde{L}^{n+1}$ and $\widetilde{\mathfrak{I}}_{0}: \tilde{S}^{n} \rightarrow \tilde{S}^{n}, \widetilde{\mathfrak{I}}_{1}: \tilde{S}^{n-1} \rightarrow \tilde{S}^{n-1}, \ldots, \widetilde{\mathfrak{I}}_{n-1}: \tilde{S}^{1} \rightarrow \tilde{S}^{1}, \widetilde{\mathfrak{I}}_{n}:$ $\tilde{S}^{0} \rightarrow \tilde{S}^{0}$ be a fuzzy foldings of $\tilde{S}^{n}$. Then we get the following chain.

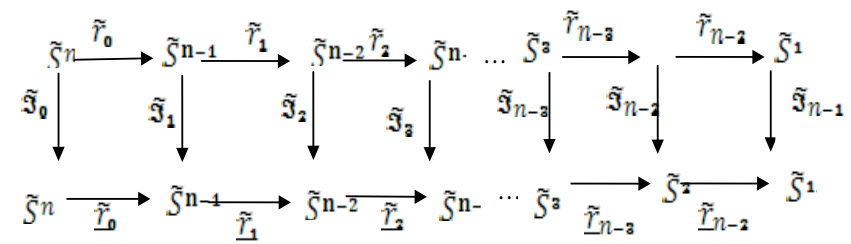

This is the general commutative diagram such that $\widetilde{\mathfrak{I}}_{i+1} \circ \tilde{r}_{i}=\tilde{\tilde{r}}_{i} \circ \widetilde{\mathfrak{I}}_{i}$.

Now, under the fuzzy central projection map $\widetilde{\beta}$, there exists a fuzzy open disk $\widetilde{D}^{n-1}\left(p, r_{1}\right) \subset \widetilde{R}^{n}$ with retractions, $\widetilde{D}^{n-2}\left(p, r_{2}\right), \widetilde{D}^{n-3}\left(p, r_{3}\right), \ldots, \widetilde{D}^{3}\left(p, r_{n-3}\right), \widetilde{D}^{2}\left(p, r_{n-2}\right), \widetilde{D}\left(p, r_{n-1}\right)$ where $r_{n-1}<r_{n-2}<r_{n-3}<\ldots<r_{3}<r_{2}<r_{1}$. Also we obtain the following chain.

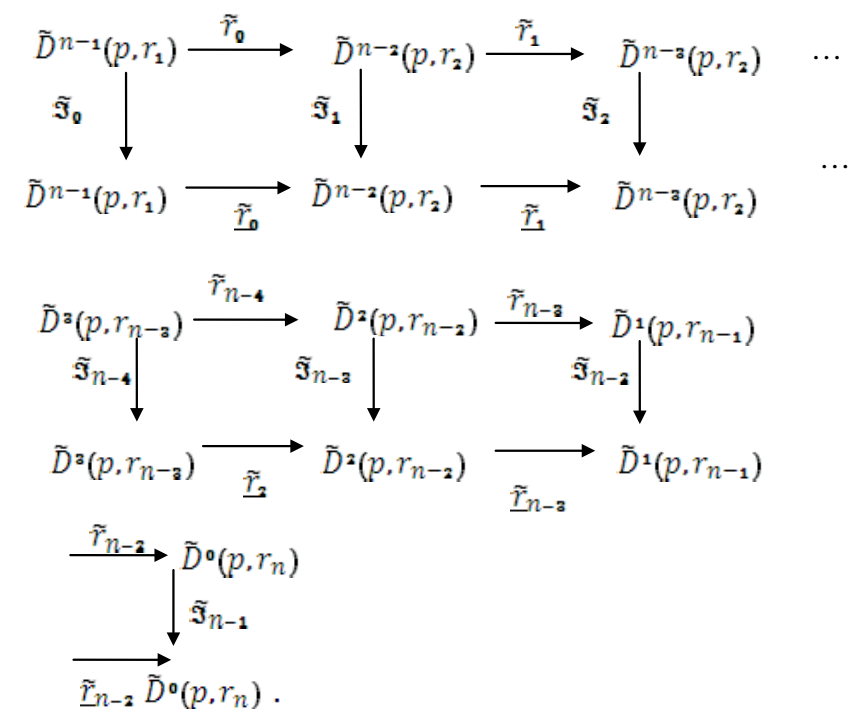

This is the general commutative diagram such that $\widetilde{\mathfrak{I}}_{i+1} \circ \tilde{r}_{i}=\underline{\tilde{r}}_{i} \circ \widetilde{\mathfrak{I}}_{i}$

TheOREM 7. If $\widetilde{\mathfrak{I}}: \tilde{S}^{n} \subset \widetilde{L}^{n+1} \rightarrow \tilde{S}^{n} \subset \widetilde{L}^{n+1}, \exp ^{-1}: \tilde{S}^{n} \rightarrow \widetilde{T}_{p}\left(\tilde{S}^{n}\right)$ and $\widetilde{\mathfrak{I}}: \widetilde{T}_{p 1}\left(\tilde{S}^{n}\right) \rightarrow \widetilde{T}_{p 1}\left(\tilde{S}^{n}\right)$, also $\tilde{\beta}: \tilde{S}^{n} \rightarrow \widetilde{D}^{n-1}$. Then $\left(\exp ^{-1}\right.$ 。 $\widetilde{\mathfrak{I}}=\widetilde{\mathfrak{I}} \circ \exp ^{-1}=\tilde{\beta}\left(\exp ^{-1} \circ \widetilde{\mathfrak{I}}=\widetilde{\mathfrak{I}} \circ \exp ^{-1}\right)$

Proof. Consider the fuzzy exponential map $\exp ^{-1}$ : $\tilde{S}^{n} \rightarrow \widetilde{T}_{p}\left(\tilde{S}^{n}\right)$, then the induced commutative diagram is defined as

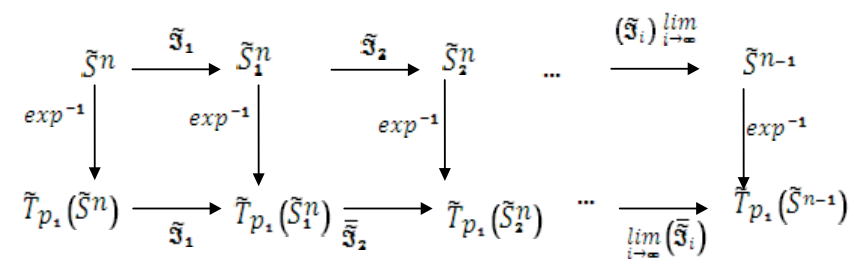


Such that $\exp ^{-1} \circ \widetilde{\mathfrak{I}}=\overline{\widetilde{\mathfrak{I}}} \circ \exp ^{-1}$.

Again, consider the fuzzy central projection map $\widetilde{\beta}$ such that $\tilde{\beta}: \tilde{S}^{n} \subset \widetilde{L}^{n+1} \rightarrow \widetilde{D}^{n-1} \subset \widetilde{R}^{n}$ and $\widetilde{\mathfrak{I}}: \widetilde{D}^{n-1} \subset \widetilde{R}^{n} \rightarrow$ $\widetilde{D}^{n-1} \subset \widetilde{R}^{n}, \widetilde{\mathfrak{I}}: T_{q_{1}}\left(\widetilde{D}^{n-1}\right) \rightarrow T_{q_{1}}\left(\widetilde{D}^{n-1}\right)$, then the induced commutative diagram is given by

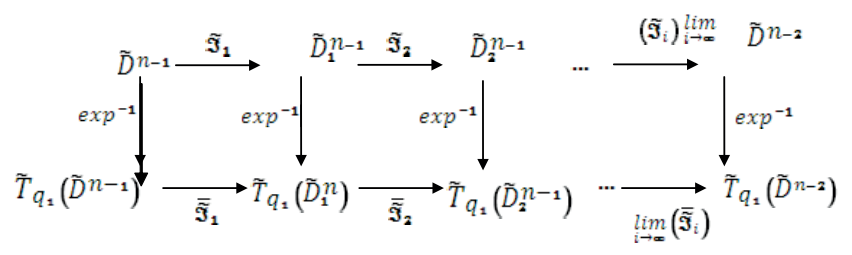

Such that $\exp ^{-1} \circ \widetilde{\mathfrak{I}}=\overline{\widetilde{\mathfrak{I}}} \circ \exp ^{-1}$.

Theorem 8. Let $\tilde{S}^{n}, \tilde{S}^{n-1}$ be two fuzzy spheres in a fuzzy Lobachevsky space $\tilde{S}^{n-1} \subset \tilde{S}^{n} \subset \tilde{L}^{n+1}$. Then any fuzzy retractions of $\left\{S^{-\uparrow} n-p\right\}$ induces fuzzy retractions of $\widetilde{T}_{p}\left(\tilde{S}^{n}\right)$ onto $\widetilde{T}_{p}\left(\tilde{S}^{n-1}\right)$. Also, under the fuzzy central projection map $\tilde{\beta}, \tilde{\beta}: \tilde{S}^{n} \subset \tilde{L}^{n+1} \rightarrow \tilde{D}^{n-1} \subset \tilde{R}^{n}$ any fuzzy retractions of $\left\{D^{-\uparrow}(n-1)-q\right\}$ induces fuzzy retractions of $\widetilde{T}_{q}\left(\tilde{D}^{n-1}\right)$ into $\tilde{T}_{q}\left(\tilde{D}^{n-2}\right)$. Then

$$
\left(\exp ^{-1} \circ \tilde{r}_{\mu}=\overline{\tilde{r}}_{\mu} \circ \exp ^{-1}\right)=\tilde{\beta}\left(\exp ^{-1} \circ \tilde{r}_{\mu}=\tilde{\tilde{R}}_{\mu} \circ \exp ^{-1}\right) \cdot \mu_{1}
$$

Proof. Consider two fuzzy spheres $\tilde{S}^{n}, \tilde{S}^{n-1}$ immersed with a common point $\mathrm{p}$. Then at $\mathrm{p}$ the fuzzy tangent spaces will be overlapped. Now, let $\overline{\tilde{r}_{\mu}}: \mu\left\{S^{\uparrow} n-p\right\} \rightarrow \mu_{\downarrow} 1\left\{S-{ }^{\uparrow}(n-1)-p\right\}$, $\mu_{1}<\mu$, be a fuzzy retractions of the fuzzy physical character of $\left\{S^{\uparrow} n-p\right\}$ into $\left\{\tilde{S}^{n-1}-p\right\}$. Then there is an induced fuzzy retractions $\overline{\tilde{r}_{\mu}}: T_{\downarrow}^{-} p \quad\left(\mu\left\{S^{-\uparrow} n-p\right\}\right) \rightarrow T_{\downarrow}^{-} p\left(\mu_{\downarrow} 1\left\{S^{-\uparrow}(n-1)-p\right\}\right)$ and $\exp ^{-1} \circ \tilde{r}_{\mu}=\overline{\tilde{r}}_{\mu} \circ \exp ^{-1}$. Now, under the fuzzy central projection map $\tilde{\beta}, \tilde{\beta}: \tilde{S}^{n} \subset \tilde{L}^{n+1} \rightarrow \tilde{D}^{n-1} \subset \tilde{R}^{n}$, let $\tilde{r}_{\mu}: \mu$ $\left\{D^{-\uparrow}(n-1)-q\right\} \rightarrow \mu_{\downarrow} 1\left\{D^{-\uparrow}(n-2)-q\right\}$ be a fuzzy retractions of the fuzzy physical character of $\left\{D^{-\uparrow}(n-1)-q\right\}$ into $\left\{\tilde{D}^{n-2}-q\right\}$. Then there is an induced fuzzy retractions $\overline{\tilde{r}}_{\mu}: T^{-} p\left(\mu\left\{D^{-\uparrow}\right.\right.$ $\left.\left.(n-1)-q\}) \rightarrow D^{-\uparrow}(n-1)-q\right\}\right) \rightarrow T_{\downarrow} p\left(\mu_{\downarrow} 1\left\{D^{-\uparrow}(n-2)-q\right\}\right)$ such that $\exp ^{-1} \circ \tilde{r}_{\mu}=\overline{\tilde{r}}_{\mu} \circ \exp ^{-1}$.

Theorem 9. Let $\tilde{S}^{n} \subset \tilde{L}^{n+1}$ be a fuzzy sphere in fuzzy Lobachevsky space which is homeomorphic to $\tilde{D}^{n-1} \subset \tilde{R}^{n}$. Then the fuzzy deformation retract of the fuzzy sphere in a fuzzy Lobachevsky space $\tilde{S}_{n}$ onto $\tilde{S}^{n-1}$ induces the fuzzy deformation retract of $\tilde{D}^{n-1}$ onto $\tilde{D}^{n-2}$.

Proof. Now we define the fuzzy deformation retract of $\tilde{S}^{n}$ as follows

$$
\tilde{F}:\left\{\left(\tilde{S}^{n}-p\right), \mu\right\} \times I \rightarrow\left\{\left(\tilde{S}^{n}-p\right), \mu\right\} \text { such that }
$$

$\tilde{F}\{(m, \mu), t\}=(1-t)(m, \mu)+t \frac{(m, \mu)}{\|m, \mu\|}$, where $\tilde{F}\{(m, \mu), 0\}=$ $(\mathrm{m}, \mu)$ and $\tilde{F}\{(m, \mu), 1\}=\frac{(m, \mu)}{\|m, \mu\|}$.

Hence, we can induce the fuzzy deformation retract of $\tilde{D}^{n-1}$ as follows

$$
\begin{aligned}
& \quad \overline{\tilde{F}}:\left\{\left(\tilde{D}^{n-1}-q\right), \mu\right\} \times I \rightarrow\left\{\left(\tilde{D}^{n-1}-q\right), \mu\right\} \text { such that } \\
& \overline{\tilde{F}}\{(x, \mu), t\}=(1-\mathrm{t})(x, \mu)+\mathrm{t} \frac{(x, \mu)}{\|x, \mu\|} \text { where } \overline{\tilde{F}}\{(x, \mu), 0\}=(x, \mu) \\
& \text { and } \overline{\tilde{F}}\{(x, \mu), 1\}=\frac{(x, \mu)}{\|x, \mu\|}
\end{aligned}
$$

Corollary 2. Any fuzzy deformation retract of $\mu$ on $\left\{S^{-\uparrow} n-p\right\}$ induces fuzzy deformation retract of $\mu$ on $\left\{D^{-\uparrow}\right.$ $(n-1)-p\}$.

Corollary 3. Any fuzzy deformation retract of $\mu$ on $\left\{S^{\uparrow} n-p\right\}$ induces fuzzy deformation retract of $T_{\downarrow} p(\mu)$ of $\left\{S^{\uparrow} n-p\right\}$

ThEOREM 10. If $\tilde{\beta}_{0}: \tilde{S}^{n} \subset \tilde{L}^{n+1} \rightarrow \tilde{D}^{n-1} \subset \tilde{R}^{n}, \tilde{\beta}_{1}: \tilde{S}^{n-1} \rightarrow$ $\tilde{D}^{n-2}, \ldots$, be a fuzzy central projection map and the fuzzy retractions of $\tilde{S}^{n}$ aregiven by $\tilde{r}_{0}: \tilde{S}^{n} \rightarrow \tilde{S}^{n-1}, \tilde{r}_{1}: \tilde{S}^{n-1} \rightarrow \tilde{S}^{n-2}, \ldots$ Then $\tilde{\beta}_{i+1} \circ \tilde{r}_{i}=\underline{\tilde{r}}_{i} \cdot \tilde{\beta}_{i}$.

Proof. Consider the fuzzy central projection map $\tilde{\beta}_{0}: \tilde{S}^{n} \subset \tilde{L}^{n+1} \rightarrow \tilde{D}^{n-1} \subset \tilde{R}^{n}, \tilde{\beta}_{1}: \tilde{S}^{n-1} \rightarrow \tilde{D}^{n-2}, \ldots, \tilde{\beta}_{n-2}: \tilde{S}^{2} \rightarrow \tilde{D}^{1}$, $\tilde{\beta}_{n-1}: \tilde{S}^{1} \rightarrow \tilde{D}^{0} \quad$ and $\quad \tilde{r}_{0}: \tilde{S}^{n} \rightarrow \tilde{S}^{n-1}, \tilde{r}_{1}: \tilde{S}^{n-1} \rightarrow \tilde{S}^{n-2}, \ldots, \tilde{r}^{n-1}$ : $\tilde{S}^{3} \rightarrow \tilde{S}^{2}, \tilde{r}_{n-2}: \tilde{S}^{2} \rightarrow \tilde{S}^{1}$. Then there are induced commutative diagrams.

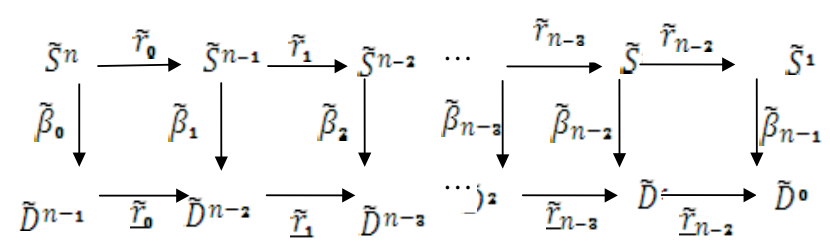

Such that $\tilde{\beta}_{i+1} \circ \tilde{r}_{j}=\underline{\tilde{r}}_{j} \circ \tilde{\beta}_{i}$.

Theorem 11. Let $\tilde{S}^{n-1} \subset \tilde{L}^{n+1}$ be a fuzzy deformation retract of $\tilde{S}^{n} \subset \tilde{L}^{n+1}$ and $\widetilde{\mathfrak{I}}: \tilde{S}^{n} \rightarrow \tilde{S}^{n}$ be a fuzzy folding

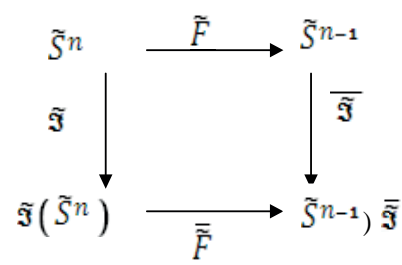


Theorem 12. If $\left(\left(\tilde{S}^{n}\right)-\{P\}\right) \tilde{r}$ is $\tilde{S}^{\mathrm{n}} \subset \tilde{L}^{n+1}$ the fuzzy retraction of $\left(\left(\tilde{S}^{n}\right)-\{P\}\right) \tilde{r}$ is $\tilde{S}^{n} \subset \tilde{L}^{n+1}$ and the fuzzy folding of $\tilde{S}^{n-1} \rightarrow\left|\left(\tilde{S}^{n}\right)-\{P\}\right|$. Then there are commutative diagram between fuzzy retraction and fuzzy folding such that

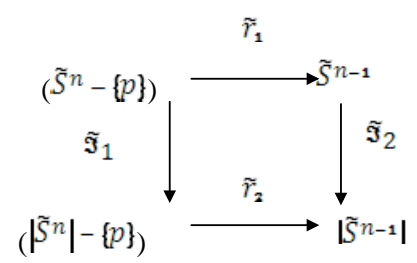

and the limit of fuzzy $\tilde{S}^{n} \subset \tilde{L}^{n+1}$

Theorem 13. The relation between the fuzzy retraction of and the limit of fuzzy folding discussed from the following commutative diagram.

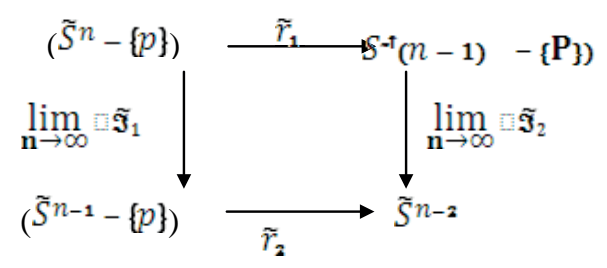

Corollary 4. The fuzzy retraction which preserves the dimension of $\tilde{S}^{n} \subset \tilde{L}^{n+1}$ is a type of the fuzzy folding.

Corollary 5. The fuzzy retraction which decreases the dimension of $\tilde{S}^{n} \subset \tilde{L}^{n+1}$ is a limit of the fuzzy folding of $\tilde{S}^{n} \subset \tilde{L}^{n+1}$.

Theorem 14. The fuzzy folding of fuzzy sphere $\tilde{S}^{n-1} \subset \tilde{L}^{n}$ are diffeomorphic to fuzzy folding of fuzzy open ball $\tilde{D}^{n-2}$ in (n-1)- dimensional Euclidean space.

Proof. Consider a fuzzy sphere $\tilde{S}^{n-1} \subset \tilde{L}^{n}$. Its fuzzification $\bigcup \overline{\tilde{S}}_{i}^{n-1}, \bigcup \underline{\tilde{S}}_{i}^{n-1}$.

Let $\tilde{f}: \cup \overline{\tilde{S}}_{i}^{n-1} \longrightarrow \cup \tilde{S}_{i}^{n-1}$ be the isofuzzy folding. Under the diffeomorphism $\gamma$ between $\tilde{L}^{n}$ and $\tilde{R}^{n-1}$, there exists a fuzzy open ball $\tilde{D}^{n-2}$ in a fuzzy $(n-1)$ dimensional Euclidean space $\tilde{R}^{n-1}$. Its fuzzification $\bigcup \tilde{\tilde{D}}_{i}^{n-2}, \cup \tilde{D}_{i}^{n-2}$ and there is an induced isofuzzsy folding $\gamma \tilde{f}: \bigcup \overline{\tilde{D}}_{i}^{n-2} \longrightarrow \bigcup \underline{\tilde{D}}_{i}^{n-2}$.

Corollary 6. Let $\cup \overline{\tilde{S}}_{i}^{n-2}, \cup \tilde{S}_{i}^{n-2} \cup \overline{\tilde{D}}_{i}^{n-2}, \cup \underline{\tilde{D}}_{i}^{n-2}$, be the upper and lower fuzzification of fuzzy sphere $\tilde{S}^{n-1}$ and fuzzy open ball $\tilde{D}^{n-2}$. If $\gamma \tilde{f}: \cup \overline{\tilde{D}}_{i}^{n-2} \longrightarrow \cup \tilde{D}_{i}^{n-2}, \forall i, j$ be a topological fuzzy folding, then there is induced topological fuzzy folding $\gamma \tilde{f}: \cup \overline{\tilde{D}}_{i}^{n-2} \longrightarrow \cup \underline{\tilde{D}}_{i}^{n-2}, \forall i, j$.

\section{Application}

1-The stream function of the acoustic gravity tripolar vortices is generalized to permit a study of the Earth's atmosphere under complex meteorological conditions, characterized by sheared horizontal flows and parabolic density and pressure profiles [7] (Figure 2).

2-Consider the flow of the fluid inside a tube [3].If we represent the velocity of the fluid as a membership degree $\mu \in[0,1]$, then $\mu=1$, in the mid of the medium where the velocity of the fluid takes a maximum and is symmetric round this line but at the edge of the tube the velocity of the fluid vanishes ,i.e., $\mu=0$.

3-The Ritz variational method [6] during the calculation of the ground - state energy in a fuzzy framework. Consider a Hamilton $H$, and an arbitrary square integrable function $\Psi$, so that $\langle\Psi / \Psi\rangle=1$. Considering $\Psi$ as a fuzzy function and the ranking system as defined in [12], similar to [6] it can be shown that $\langle\Psi / H / \Psi\rangle$ is a fuzzy upper bound on $E_{0}$ (ground-stat energy). Now $\langle\Psi / H / \Psi\rangle$ should be minimizing the distance between $E_{0}$ and respect to a number of parameters $\left(\alpha_{1}, \alpha_{2}, \ldots\right)$. This can be done by minimizing distance between $E_{0}$ and $\langle\Psi / H / \Psi\rangle$. The rest of the discussion is the same as that provided in [6].

\section{Conclusion}

In the present paper, we obtain and study some types of fuzzy retractions of fuzzy open sphere in fuzzy Lobachevsky space. Also, we deduced some types of fuzzy deformation retract of fuzzy open sphere. The relations between the fuzzy

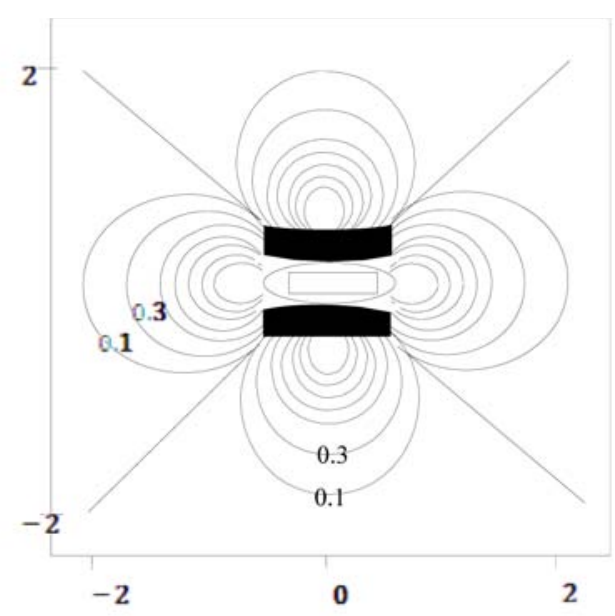

Figure 2. Application of fuzzy manifold. 
folding and the fuzzy deformation retracts of fuzzy open sphere are obtained. Some applications are presented.

\section{Acknowledgements}

The author is deeply indebted to the team work at the deanship of the scientific research, Taibah University for their valuable help and critical guidance and for facilitating many administrative procedures. This research work was financed supported by Grant no. 3066/1434 from the deanship of the scientific research at Taibah University, Al-Madinah Al-Munawwarah, Saudi Arabia.

\section{References}

1. El-Ahmady A E (2007). The variation of the density on chaotic spheres in chaotic space-like Minkowski space time, Chaos, Solitons \& Fractals, vol 31(5), 1272-1278.

2. El-Ahmady A E (2007). Folding of fuzzy hypertori and their retractions, Proceedings of the Mathematical and Physical Society of Egypt, vol 85(1), 1-10.

3. El-Ahmady A E (2006). Limits of fuzzy retractions of fuzzy hyperspheres and their foldings, Tamkang Journal of Mathematics, vol 37(1), 47-55.

4. El-Ahmady A E (Accepted). Fuzzy elastic Klein bottle and its retractions, International Journal of Applied Mathematics and Statistics.

5. El-Ahmady A E (2012). Folding and unfolding of chaotic spheres in chaotic space-like Minkowski space-time, The Scientific Journal of Applied Research, vol 1(2), 34-43.

6. El-Ahmady A E, and El-Araby A (2010). On fuzzy spheres in fuzzy Minkowski space, Nuovo Cimento, vol 125B(10), 1153-1160.

7. El-Ahmady A E (2011). Retraction of chaotic black hole, The Journal of Fuzzy Mathematics, vol 19(4), 833-838.

8. El-Ahmady A E (2012). Retraction of null helix in Minkowski 3-space, The Scientific Journal of Applied Research, vol 1(2), 28-33.

9. El-Ahmady A E (2004). Fuzzy Lobachevskian space and its folding, The Journal of Fuzzy Mathematics, vol 12(2), 609-614.

10. El-Ahmady A E (2004). Fuzzy folding of fuzzy horocycle, Circolo Matematico di Palermo, Serie II , Tomo L III, 443-450.

11. El-Ahmady A E, and Shamara H M (2001). Fuzzy deformation retracts of fuzzy horospheres, Indian Journal of Pure and Applied Mathematics, vol 32(10), 1501-1506.
12. El-Ahmady A E, and Al-Luhaybi A S (2013). Fuzzy retractions of fuzzy open flat Robertson-Walker space, Advances in Fuzzy Systems, vol 2013.

13. El-Ahmady A E, and Al-Onemi K (2013). On the folding of Lobachevsky space, International Journal of Applied Mathematics and Statistics, vol 4(10), 13-23.

14. El-Ahmady A E (2011). The geodesic deformation retract of Klein bottle and its folding, The International Journal of Nonlinear Science, vol 9(3), 1-8.

15. El-Ahmady A E (2013). On the fundamental group and folding of Klein bottle, International Journal of Applied Mathematics and Statistics, vol 37(6), 56-64.

16. El-Ahmady A E (2013). Folding and fundamental groups of Buchdahi space, Indian Journal of Science and Technology, vol 6(1), 3940-3945.

17. El-Ahmady A E, and Al-Hazmi N (2013). Foldings and deformation retractions of hypercylinder, Indian Journal of Science and Technology, vol 6(2), 4084-4093.

18. El-Ahmady A E, and Al-Luhaybi A S (Accepted). On fuzzy retracts of fuzzy closed flat Roberstion-Walker spaces, Advances in fuzzy sets and systems.

19. El-Ahmady A E, and Al-Luhaybi A S (2013). Retractions of fuzzy flat Robertson-Walker space, International Journal of Applied Mathematics and Statistics, vol 41(11), 116-129.

20. Schmeikal B (2010). Primordial Space -The Metric Case, Chapter 1, New York.

21. Naber G L (2011). Topology, Geometry and Gauge fields, Chapter 1, Springer-Verlage New York, Berlin.

22. Hartle J B (2003). Gravity, An introduction to Einstein's general relativity, Chapter 3, Addison-Wesley, New York .

23. Griffiths J B, and Podolsky J I (2009). Exact Space-Times in Einstein's General Relativity, Cambridge University Press, Cambridge, New York.

24. Zadeh L A (1975). Fuzzy sets and their application to congnitive and decision, Chapter 1, New York, Academic Press.

25. Reid M, and Szendroi B (2005). Topology and Geometry, Chapter 1, Cambridge University Press, Cambridge, New York.

26. Arkowitz M (2011). Introduction to homotopy theory, Chapter 1, Springer- Verlage, New York.

27. Palaniappan N (2002). Fuzzy Topology, Chapter 1, London, New York

28. Straumann N (2004). General relativity with application to astrophysics, Chapter 4, Springer-Verlage, New York, Heidelberg, Berlin.

29. Strom J (2011). Modern classical homotopy theory, Chapter 1, American Mathematical Society.

30. Kudryashov V V, Kurochkin Y A (2010). Motion caused by magnetic field in Lobachevsky space, arXiv :1006. 5202vI [math-ph], vol 1205, 108-111. 